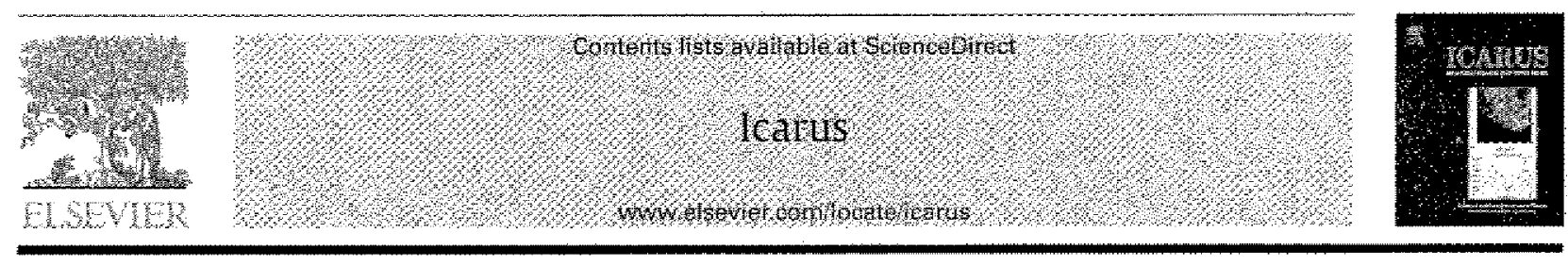

\title{
THEMIS observations of Mars aerosol optical depth from 2002-2008
}

\author{
Michael D. Smith \\ NASA Godadod Space Fligft Center. Greenbelt. MO 20\%?. USA
}

\section{$A R T C L$ I $\mathrm{NFO}$}

\section{Article fistory:}

Reteived 23 Janary 2009

Recised 18 March 2009

Aecepted 21 ifarch 2009

Avaluble otalite 25 warcl 2009

Reyword:

Mirs. atmosphent

Mars, ciminate

Atmospheres, structare

\begin{abstract}
$A$ B 5 T A C T
We use infrated images obtained by the Thema Etricsion Imagitg System (THEMIS) instrument on. boatd Mars Odyssey to retrieve the optical septh of dust and watef lce aerosols over mote than 3.5 martian years between february 2002 (MY $25, L_{s}=330^{2}$, and December 2008 (MY $29 . \mathrm{L}_{\mathrm{s}}=183^{\circ}$. These data provide an important brige between eatler tes observations and recent observations fron Mars express and Mars Reconnaissance Orbiter. An inprovement to our earlier retrieval |Snth, M.D.

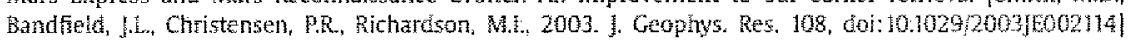
to indude atmospheric temperature information fom THEMIS Band 10 observations leads to much frhproved retrevals duting the largest dist storms. The new retrievals show moderate dust storm activity during Mars Years 26 and 27 , althotigh details of the strength and timing of dust storms is different from year to year. A platetencirching dust stom event was observed durng Mars Year 28 near Southern Hemisphere Summe solstice. A bett of lowntitude water foe clouds was observed during the aphelion season duritu zach year, Mar's Years 26 though 29 . The optical depth of water ice clouds is somewhat higher in the THEMS retrievas at $5,500 \mathrm{PM}$ focal the thats in the TES retrievas at $-2,00 \mathrm{PM}$, suggestive of posstble tocal tine vartation of clouts.
\end{abstract}

Published by Elsevier inc.

\section{Introduction}

The continued successful aperation of the Themal Fnission Imaging System (THEMS) instrument on-board the Mars Odyssey spaceciaft has allowed for the long-term monitoring of the Mars atnosphere for nore that 3.5 martian years (Christensen et at. 2003). The atmosphere has been studied using both the visible and the infared portions of the THEMIS instrument. Themal infrared observations made by THEMIS in nine spectral bands enable the fetrieval of the spatial and seasonal variation of cust and water ice columb-integrated aerosol optical depth, as went as surface temperature and a vertically-averaged atmospheric temperature (Smith et al, 2003). THEMIS visible images have been tused to characterize the morphology and seasonal dependence of dust and ice clouds (Inada et al., 2007), and to charaterize mesoscale clouds (MeConnochie et al., 2006).

The fetrievals of aerosol optical depth from THEMIS observations provide a tratid link between the observations of aerosols taken by the Mars Global Surveyor TES (e.g. Smith, 2004) and Mars obiter Camera (MoC) instruments (e.g. Cantot et at. 2007; Cantor; 2007) and those taken by instruments on-board the currentlyoperating Mars Reconnaissance Orbiter (MRo) (e.g. Wolf et al. 2009) and Mats Express (MEx) orbiter (e.g. Zasova et al.r 2005; Rannou et ah, 2006). The long overap in time (more than one matian year) between the THEMIS observations and both MGS

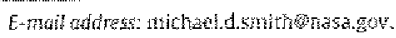

and MROFMex observations provides an oppotunity for the validation and cross-calibration between all the different instruments. Together, these datasets now cover a period of more than five martian years, enabling the initial exploration of interannual variations in atmospheric conditions.

The retrieval of dust and water ice aerosol optical depth using THEMIS infrared observations was previously descrbed by Smith et al. (2003), and mote information on the THEMIS instrument can be found in Chrstensen et al. (2003). A recent review of all previous spacecant observations of aerosols in the martian atmosphere is atso given by Smith (2008). In this paper. we describe improvements fo the Smith el al. (2003) retrieval as well as results updated to the current time. In Section 2 we describe the set of THEMIS observations used in the retrieval of aerosol optical depth. In Section 3 we describe the retrieval alyonithm, focusing on the improvements that have been made to the Smith et al. (2003) algorithm in Section 4 we present the results of the retrieval along with a discussion of results, and we summarize our mindings in Section 5

\section{Data set}

\section{THEMIS insttument}

The THEMIS instrument obtains images of Mars assing one of two separate focal planes. One focal plane contains ten spectral fil. ters coventug the thermal infrared between 6.5 and 15 um, while 


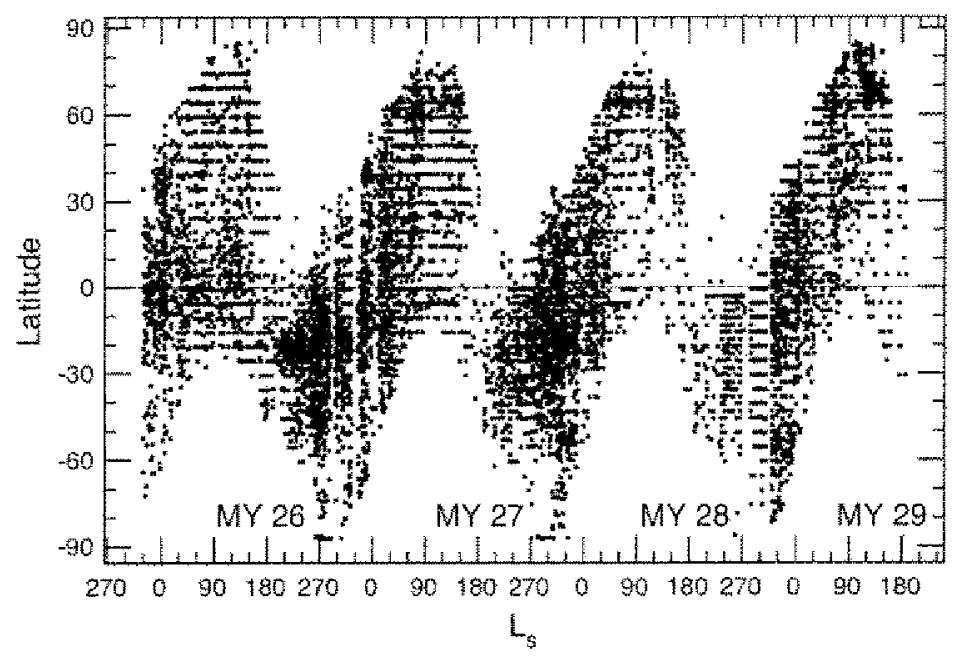

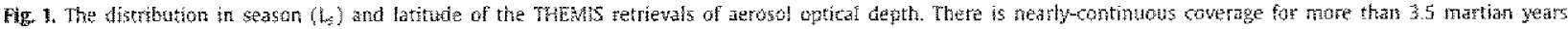
Smeth gaps in coverage are caused by solar conjuntion and syaceraft atomales.

the other contains five spectral flters at visible wavelengths be. tween 0.45 and $0.85 \mathrm{um}$ (Christensen et at. 2003). in this work we use oniy the images taken in the thermal infrared. The first two THEMIs spectal bands are identical and are centered at $6.78 \mathrm{um}$. Bands 3 through 10 are centered at $7.93,8.56,9.35,1021$, $11,04,11,79,12.57$, and 14.88 , 1m, fespectively, with spectral widths that allow for slight overlaps between adjacent bands (except for band 10. which is somewhat more narrow and has no spectral overtap with any other band,

The THEMS inages are 320 pixels across-track and are buit up in the along-track direction over time by spacecraft motion. Images ate of variable length depending on the purpose of the observation and the available resotaces avalable for downlink of the data. The size of a THEMIS pixel at the surface is $100 \mathrm{~m}$. The locat time of the THEMts observations varies between roughly $4: 00$ and $6: 00 \mathrm{PM}$. which is somewhat later than either the 2 :50 PM mean lotal time of Mars Global Surveyor or the 3:00 PM mean local time of the Mars Reconnaissance Orbiter.

We are most interested in the variations of atmospheric conditions on scales larger than the THEMIS pixel size, so for each THEMLS image we use as our observation the average of a block of data 320 pixels wide (the entire widt of the image) by 256 pixels long. The tast 256 pixels of each innage are used for this average because they have the most accurate calibration (an effect only now ticeabte for Band 10). The resulting average covers an area 32 by $26 \mathrm{~km}$ in size, or roughly one half degree square.

\section{Observations thed in this study}

THEM1S images are divided between those targeted at objects of geologic interest, general mapping of the entire surface, and a latitude-longitude grid of observations taken every couple weeks intended to survey atmospleric conditions. Although the targeted observations generally provide a feasonable global-scale sampling over time, the atmospheric grid has proven to be very useftul as a means to "nill in" any spatial gaps in the coverage of targeted observations

Included in this work are all THEMS images that include all ten infrared bands and for which the surface is sufficiently warm $(220 \mathrm{~K}$ or higher) to provide enough thernal contrast between surface and atmosphere to allow for a reliable retrieval. A total of 26,400 images fit these criteria, covering the period from the beginning of the mission in February 2002 , or Mars Year (MV)
25. $L_{5}=330^{\circ}$, to the end of December 2008 (MY 29, $\mathrm{L}_{5}=183^{\circ}$ ) Athough the number of avalable inages. per day varies sonewhat with time, the coverage is nearly continuots over these 3.5 martian years with only short interruptions caused by solar conjunction or spacecraft anomales.

Fig. 1 shows the seasonal $\left(L_{c}\right)$ and latitudinal coverage of the THEMIS infrared images used in this work. Atthough the THEMIS instrument camot provide a continuous pole-to-pole mapping because of data rate constraints, the coverage is still more than 5 to ficient to provide a valuable picture of the seasonal and spatial trends of atmospheric aerosol optical depth. In particular, the vatue of the atmosoneric grids can easily be seen during the aphation season $\left(\mathrm{L}_{\mathrm{s}}-\mathrm{J}^{\circ}-180^{\circ}\right)$ when data rates have typically been low

\section{Revised retrieval atgorithm}

The retrieval used here is the same as Smith et al (2003) atgofithm, except modifed to include atmospheric temperature infor. mation from the THEMIS observations themsefves. Here we give a brtef outline of Snith et al. (2003) algotithm, and in the next section we describe the revision to include temperature intomation from the Thims observations.

The basic idea of the THEMIS retrieval is to find the values for surface temperature and dust and water ice aetosol optical depth that provide the best fit between the computed and observed raGarce spectra in THEMS Bands $3+8$ (roughy $8-12$ Hm). The three Guantites, surface temperatute, dust optical depth, and water lee optical depth are fit simultaneously by linearizing the solution for radiance about the current best gtess and iterating until solution. Convergenee typically ocours in less than hwe iterations.

Radiance is computed using a purely absorbing plane-paraltel atmosphere. Thus, the opticat depth values teported here shoutd be considered as effective absorptive optical depth only, not fult extinction optical depth that includes scattering. Numerical experiments show that extincion optical depth is roughy 1.3 times absorption optical cepth for dust, and 1.5 times absorption optical depht for water ice aerosol (Smith, 2004).

The spectral dependence of optical depth for dust and water ice aerosols is taken from TES retrievals (Bandfield and Smith, 2003) and are assumed to be constant in space and time. Because surface emissivity has a spectral dependence that is very similar to that of dust aerosol pver the THEMIS wavelengthe, the two cannot be retreved independently. Instead. TES festats are used for the spectral 

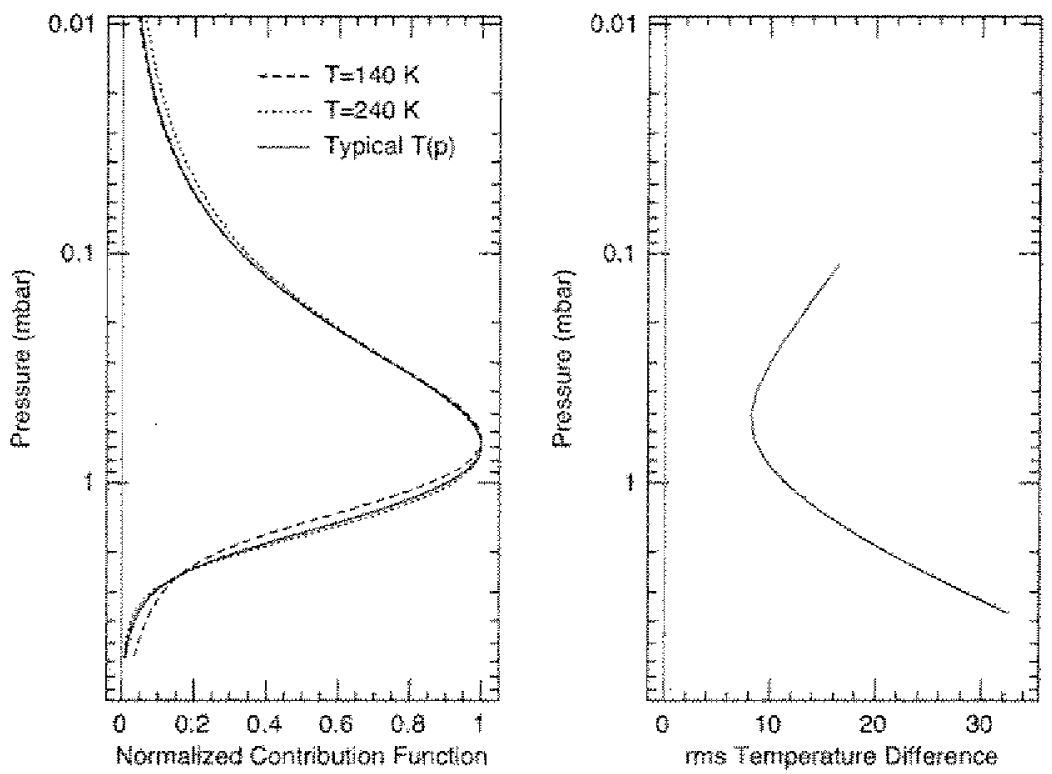

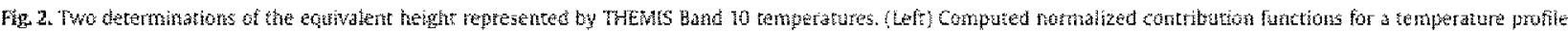

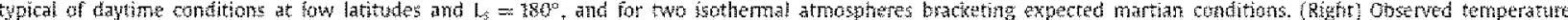

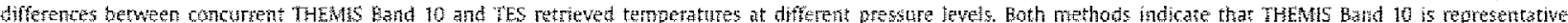
af a wide range of hetghts centered at about 0,5 mba:

dependence of surface emissivity (Bandfeld and Snith, 2003) and for the amplitude of surface emissivity as a function of latitude and longitude (Smith, 2004).

The vertical distribution of dast aerosol is asstmed to be well. mixed. Water ice aerosol is assumed to be in the form of condersate clotds, so that there is no aerosol betow the water condensathon tevel. The condensation level is computed using a water vapor abindance taken from concurrent TES observations, or from TES climatology after the end of systematic TES spectrometer observa. tions $\left(\mathrm{MY} 27, \mathrm{~L}_{5}=81^{\circ}\right)$

\section{Indusion of THEMIS temperature information}

The one THEMIS band sensitive to atnosphetic tenperature (Band 10) was designed to give a temperature representative of $a$ broad range of the atmosphere (roughly $0.1-2.0 \mathrm{mbar}$ ) similits to the "TH" temperature derived from Viking Orbiter IRTM observattens (Martin and Keffer, 1979). Althotgh usefu, this single vetticaly-integrated temperature by itself is not sufficht to acturately nodel atmospheric radiance, The Smith et al. (2003) atgorithm relied on concurrent retrievals from TES to provicte the necessary temperature profiles. That strategy is no longer possible after the fallure of the TES spectrometer (near-continuous obserm vations by the TES spectrometer ended on 31 August 2004, or MY $27, L=81^{\circ}$ ). For more recent THEMIS obserwations we use a combination of the averaged atmospheric temperature proviced by THEMS Band 10 with historic observations by TES at the same season and location (i.e climatology) to estimate the temperature profle, $T(p)$ at the time of each THEMIS obsevation.

Althos the temperature derived from THEMS band 10 is representative of a broad vertical range of the atmosphere, it is useftu to define a single effective height for this temperature. Fis. 2 shows both a theoretical and an empirical derivation of this height. The teft panel of Fig 2 shows the contribution function for THEMIS Band 10 computed by taking the vertical derivative of the transmititance of the $\mathrm{CO}_{2}$ atmosphere as a function of height integratad over the spectral response of THEML Eand 10. The contritution function shows the vertical distribution of the ratave contribu tion to the obseryed radiance in THEMIS Band 10. This distribution is not sensitive to the temperature profle as shown by the very similar contribution functions for three widely different tempera ture profiles. The peak of the contribution function is at a pressure level of about 0.6 mbar, although the peak is broad. The right panet of fig. 2 shows the rms temperatute difference between observed THEMIS Fand to brightness temperatures and the temperatures at differetu pressure levels extracted from concurrent TES retrievals. This comparison indudes all THEMIS observations taken during the period when the TES spectrometer was still operationat, and so includes a complete range of seasons and latitudes. The minimum temperature difference is found at a pressure level of 0.5 mbar, which is in reasonable agreenent with the theoretical results given the wide vertical distribution apparent from both methods. For the putpose of this retreval, we assign an effective height of 0.5 mbar to the THEMS retrievats.

Much of the time using TES chmatology temperatures tor the THEMS retrieval is a reasenable approximation, Fig. 3 shows a comparison between TES clmatology temperatutes at $0.5 \mathrm{mbar}$ and observed THEMIS Band 10 temperatures. The TES climatology is taken from the first martian year of TES operations, from $M Y 24, L_{s}=104^{\circ}$ to $M Y 25, L_{5}=104^{\circ}$. There is excellent correspondence beween the two during the aphelion season, $\mathrm{L}_{s}=0^{\circ}$ to $180^{\circ}$. However, it is also apparent that the randont timing and strength of large regional and planetary-scale dust stoms during the perthelion season, $L_{s}=180^{\circ}$ to $360^{\circ}$, makes the use of TES tilmatology at those times much less desirable. These temperature differences can be onite large, exceeding $30 \mathrm{~K}$ for the langest dust storms. When actual atmospheric temperatures ate greater than those predicted by TES clmatology, then the thermal contuast between the surface and atmosphere will be overestimated, which means that aerosol optical depth will be underestimated.

It was noted by Snith (2004) that the atmosphentic warming caused by dust stoms varies witt height. The largest response is at high altitudes, while there is little response at hevels near the surface. Fog 4 shows a time series of temperatures at low Southern latitudes trom TES over three martian years for fue different atmow sphetic levels. While there ss laroe onterannual variation from ycar 


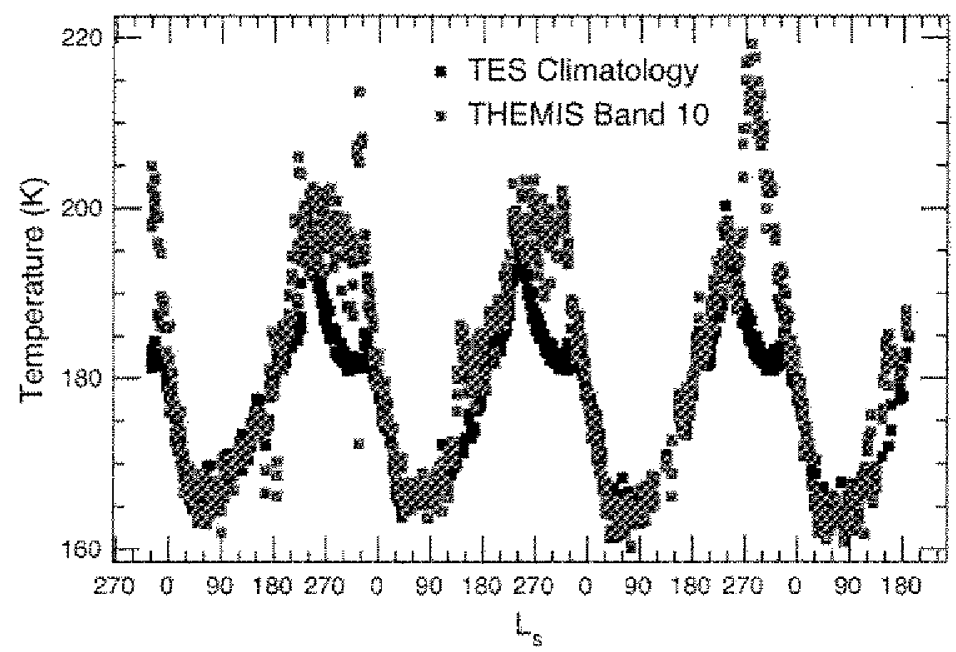

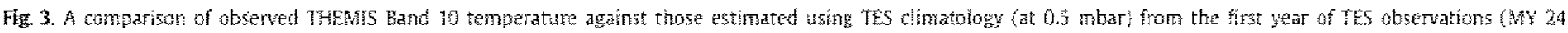
and 25) IES chimatology provites a good extinate except when there are large dust stoms.

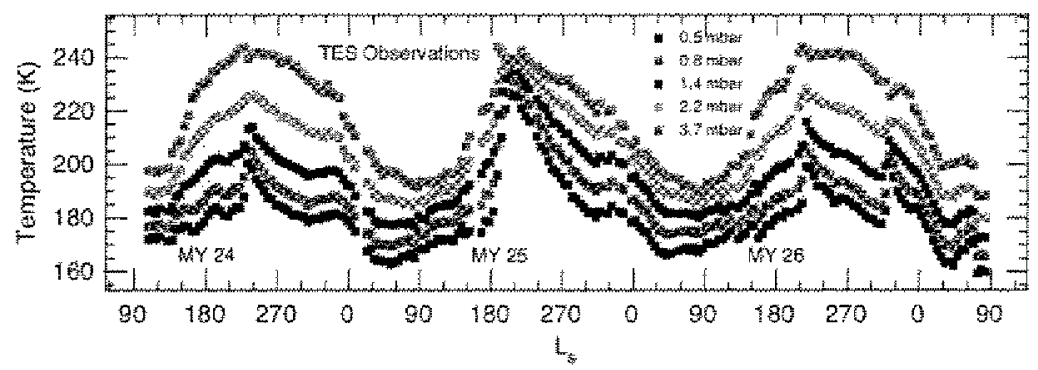

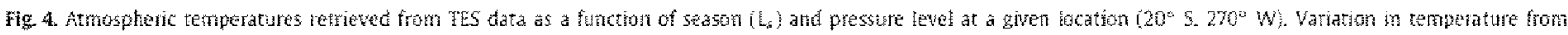

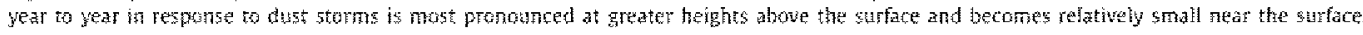

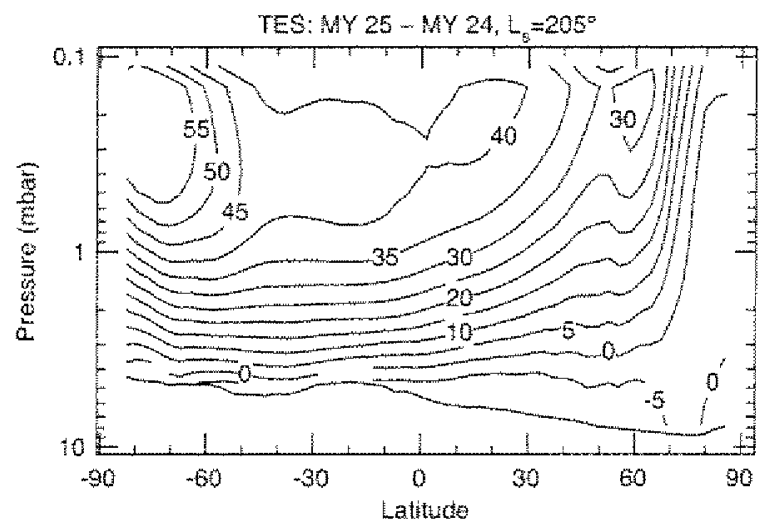

Fig. 5. The difference in zonaly-avefaged thermat structure of the Tratian atmo-

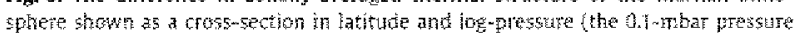

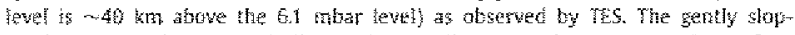

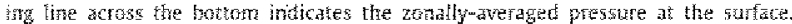

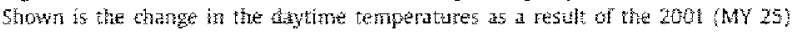

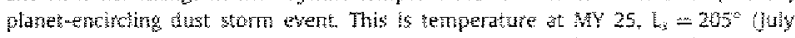

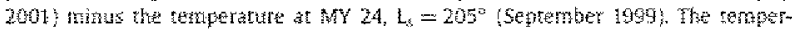
anure differene rises almost linearly from near-zero at the surface to a maximum value somethere above the 0.5 -moth lewel.

to year caused by cust storns at 0.5 mbar, the year-to-year variation near the surface $(e, g, 3.7$ mbar $)$ is much reduced. Another example is given by fig. 5 , which shows the temperature differ- ence as a function of iatitude and height between temperatures observed by TES at MY 25, $\mathrm{L}_{s}=205^{\circ}$ at the peak of a planetencircling dust stom and those observed exactly one martian year previousiy when there was no dust storm (Smith et al., 2002). Here we see a nearwero tempenature change at the suface increasing roughly lineatly with height.

The TES observatons of temperatures curing dust storms suggest a relatively simple correction based on THEMIS Band 10 temperatures that can be made to improve the estimate of the tem peratwe profle from TES cimatology. We define a quartity, $A T$. as the difference between the observed THEMIS Band 10 temperature and the TES climatology temperature at $0.5 \mathrm{mbar}$ at the seasof, latitude, and longitude of the THEMS observation). Recalling Fig. 3 , most of the time $\Delta T$ is within $3 \mathrm{~K}$ of zero, but during dust storms $\Delta T$ becones large and positive with values of $10-40 \mathrm{~K}$. The temperature profile at the time of the THEMIS observation. $T(p)$, is then estimated from the TES climatology temperature pofte, TTES (p), using:

$T(p)=T_{\mathrm{S} S}(p)+0.4 \lambda T \log \frac{p_{\operatorname{me}}}{p}$

Where pref is a reference pressure level taken to be 6.1 mbar. Equation (1) provides a temperature correction that is zero at the reference pressure (essentially the suface) and increases linearly in log-pressure (neary linear in height) to a value of $\Delta \vec{i}$ at a presstife fevel of 0.5 mbar. 

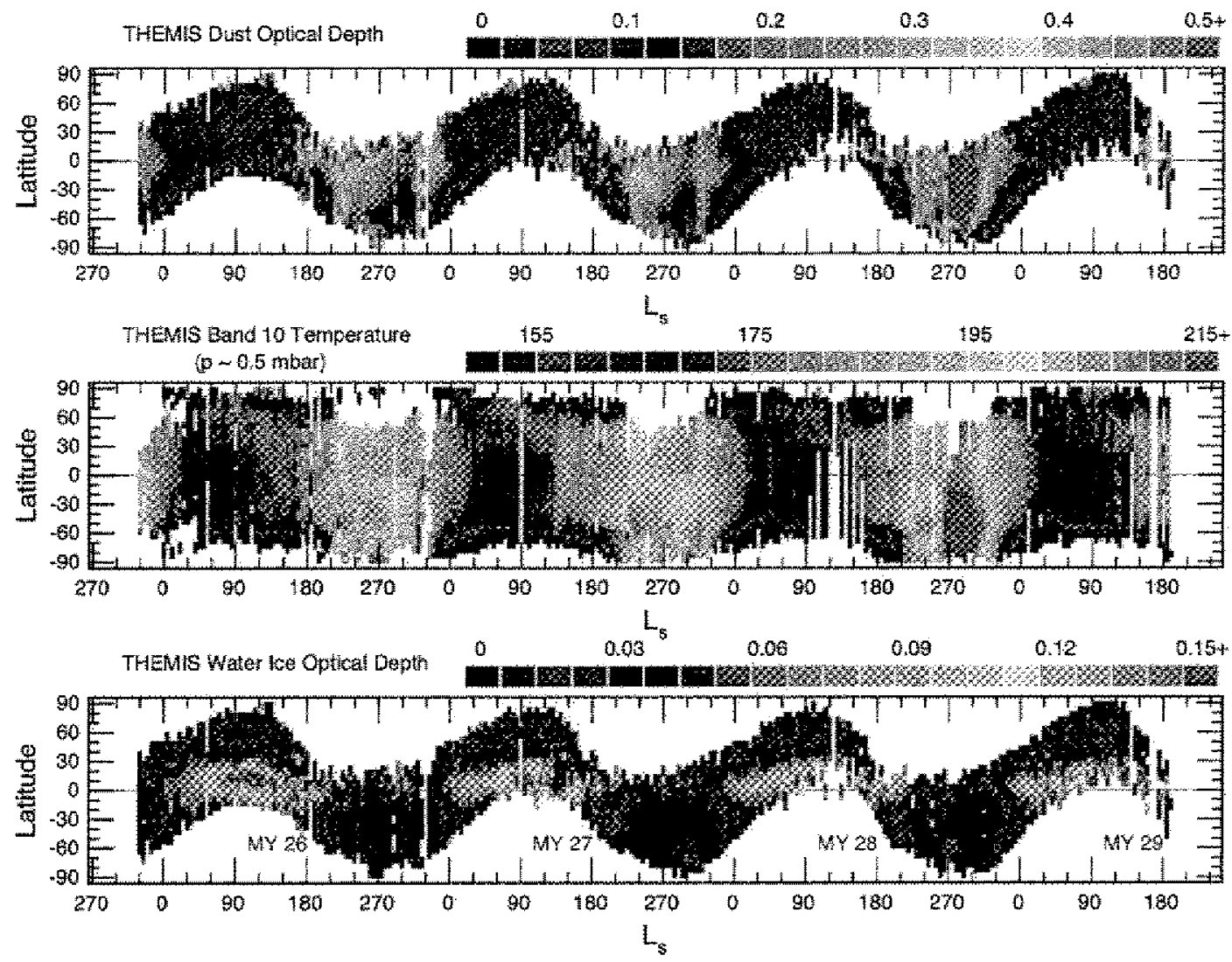

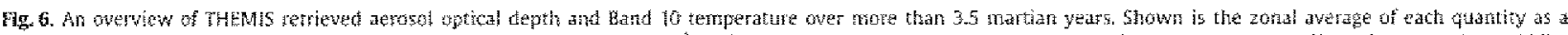

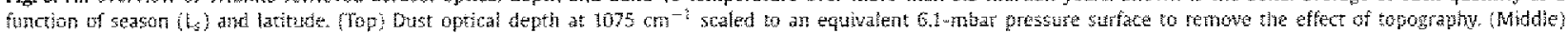

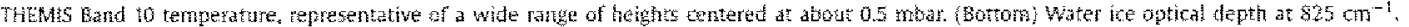

\section{Estimation of uncertainties}

A number of sources contribute to ancertainties in the retrieved values of dust and water ice opticat deptin, including instrument noise and calibration, frror in the assumed temperature profle and surface emissivity, and simplifying assumptions of fixed spectra! shapes and a well-mixed non-scattering aerosol. The formal propagation of random instrument noise leads to negligible error since we average over $320 \times 256$ pixel frames. Uncertainty from calibation and other systematic errors is not reduced by averaging pixels. Such uncertainties are diffcult to evaluate, but our experience with the TES retrievals and the comparison of THEMIS optical depth results with JES leads to an estimate of an uncertainty for a single THEMIS observation of 0.04 or $10 \%$ of the total absotption optical depth, whichever is Jarger. Uncertainties are likely somewhat higher (perhaps $20 \%$ or even greater) during the most intense dust stoms becatse large corrections to the temperature profle must be made for those observations. The estimate for the uncertainty in optical depth also does not indude the systematic difference Getween absorption and extinction optical depth. As stated eallier, we estimate that the extinction optical depth (including scattering) is systematicaly higher than the absorption optical depth reported here by $\sim 30 \%$ for dust and $\sim 50 \%$ for water ice.

\section{Results}

The retrieval of dust and water ice aerosol optical depth was peformed on all THEMIS daytime infrared inates with surface temperature greater than $220 \mathrm{~K}$ and all ten spectral bats present.
The images span the antire 3.5 martian years of Mars Odyssey op erations fom $M Y 25, L=330^{\circ}$ (19 February 2002) to the present, MY $29, L_{5}=183^{\circ}$ (31 December 2008), yielding a total of 26,400 good refrievals,

Fig. 6 shows the restits of our retrievals. Because dust has been assumed to be well-rinixed, in Fig. 6 and the following figutes we present dust optical depth scaled to an equivalent 6.1-mbar pressure surface to renove the effect of topograpty. This is accomplished by multiplying dust optical depth by the factor (6.1 mbatisufface pressure). Water ice acrosols have not been assumee to be well-mixed, and so we present water ice optical depth without any scaling. Band 10 temperatures are stown for all daytime infrared images without the restriction on surface temperature.

\subsection{Dust optcat depth}

The history of dust optical depth shows the familiar seasonal dependence observed by previous spacecaft (Smith, 2008). The highest dust optica depth on a global scale is found during the perihelion season $\left(L_{s}=180^{\circ}-360^{\circ}\right)$, but with significant variation in the tiring and ampltude of large dust stoms from one martian year to the next. Relatively low dust optical depth persists throughont most of the aphelion season with the exception of occasional small-scale dust stoms at high latitudes along the edge of the refreating polar ice caps.

The MY 26 dust storm season was relatively mild. As usua!, dust activity began to pick up after $L_{s}=140^{\circ}$ with the frist large regional storms at around $L_{s}=210^{\circ}$. Additional dust activity was 
observed at high southern latitudes near sumner solstice. The dust stoms at $L=315^{\circ}$ are a regular feature of the dust cycle, but were noticeably more intense than those seen in previous years at that season (Lu et al., 2003; Smitt, 2004). These dust storms were particulaty notable since they occurred at the time of the Beagle 2 arrival at Mars, and just prior to the landings of two Mars Exploration Rovers (MER)

The MY 27 dust stom season was also mild, although there were several differences in the timing and intensity of the largest dust storm events compared with $M \times 26$. An unusually early and intense low-latitude dust stom was observed in $M Y 27$ at $\mathrm{L}_{\mathrm{s}}=135^{\circ}$, which was atso observed at both MER landing sites by the rovers (Smith et al, 2006). However, this early attivity was not followed up by increased dust optical depth during the rest of the season. Regionat dust storms at $L_{s}=225^{\circ}$ were comparable in nntensity although later than those observed in MY 26. The tateseason dust activity at $L_{5}=310^{\circ}$ was more similar to the moderate Etast storms observed in MY 24 and 25 by TES (Liu et al., 2003: Smith, 2004) than the more intense dust event of MY 26.

By far, the greatest dust activity observed so far by THEMIS cecurred buting MY 28 . The early-season dust activity was not exceptional, with the regional dust activity at $\mathrm{L}_{s}=220^{\circ}$ somewhat less intense than in previous years. However, at $L_{s}=265^{\circ}$ a seties of dust storms began, which lead to a planet-encircling dust event. Thermal infrared dust optical depth exceeded unity over most of the planet for several weeks making this the highest globat cust loading since the MY 25 (2001) event (Smith et al. 2002; Cantor, 2007). Dust optical depth only gradually decayed, staying above 0.5 until at least $t_{s}=310^{\circ}$. No new significant dust activity was observed by THEMIS during the usual $\mathrm{E}_{\mathrm{s}}=310^{\circ}-320^{\circ}$ dust stom period, ptesumably because of the large perturbations of conditions from the late-season planet-enciring dust event.

Observations from THEMIS as we enter the dusty season for MY 29 once again slow elevated early-season dust activity similar to that observed in MY 27 .

\subsection{THEMS Band 10 temperatures}

The micdle panel of Fig. 6 shows THEMIS Band 10 brightness temperature, which was shown above in Section 3.1 to be a broad vertical average of atmospheric temperature with an effec tive height of rosghly 0.5 mbar or $25 \mathrm{~km}$ above the suface. Atmospheric temperatures respond to a combination of orbital (perihelion us. aphelion) and seasonal (summer vs. winter) variations, as well as to direct heating by the absorption of suntught by dest and to general cinctation pattems. The observed heatirg caused by the large regional storms duing MY 26 and 27 was roughty $15-20 \mathrm{~K}$, while that catsed by the planet-encircting dust event of MY 28 was $30-40 \mathrm{~K}$. Globally-averaged temperatures have a con sistent and weild defined minimum near $L_{s}=40^{\circ}$, which is earler than both aphetion $\left(L_{s}=71^{\circ}\right)$ and the global minimum dust opti cal depth $\left(L_{s}=130^{\circ}\right)$, phenomenon also observed by TES (Smith, 2004).

\subsection{Water ice optical depth}

The main feature evident in the THENis retrievals of water lice aerosol optical depth (bottom pane of Fig. 6 ) is the aphelion season low-latitude doud belt. This robust set of clotad features has been observed repeatedy by telescopic observatons (e.g. Cincy et a[, 1996), Vking (e.g. Tamppari et at, 2000), TES (peari et al., 2001; Lit et al. 2003: Smith. 2004), and other instruments. Low-latitude water ite optical depth increases tapidiy after $L_{s}=0^{\circ}$ reaching its peak value by about $L_{n}=60^{\circ}$. The cloud belt begins to dissipate after $L_{s}=140^{\circ}$ as atmospheric temperatures inse, but remnants persist until at least $L_{s}=180^{\circ}$. At its peak, there is signifcant doud

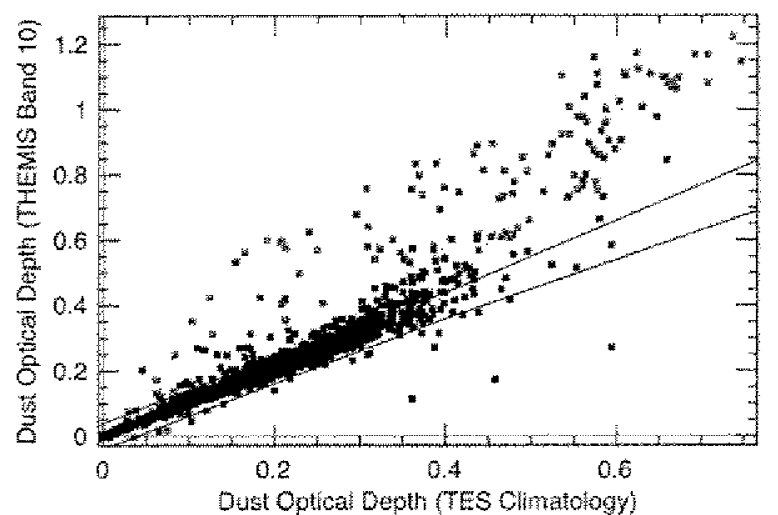

Fig. 7 A conpazison of retrevals of dust optical depth from THEMS obserwation

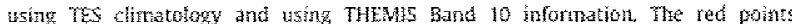

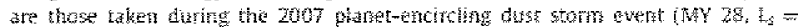
$265^{*}-305 !$

cover between $10^{\circ} \mathrm{S}$ latitude and $30^{\circ} \mathrm{N}$ latitude, with enhancements over ateas of elevated topography such as Elysium, Tharsis. Olympus Mons, and Alba Patera. There is relatively ittle interannual variation in the timing, location, and amplitude of the cloud belt However, the THEMS observations to show that very eary low-latitude dust storms, such as at MY $27, \mathrm{~L}_{s}=135^{\circ}$, can very quickly reduce cloud optical depth to near-zero valnes. In this particulat case, the cloud belt partially zeformed by $L_{s}=150^{\circ}$ alter the dissipation of the dust stom and atmospheric conditions had returned to nominal values for that season.

The polar hood douds that form at high latiudes in the winter hemisphere are not observed by THEMIS because at the relatively late local time of the Mars Odyssey orbit $(\sim 5: 00 \mathrm{PM})$ surface temperatures are too low at those latitudes to provide the thermal contrast between the surface and atmosphere necessary for the reliable retrieval of water ice cloud optical depth.

\subsection{Comparison against retreval using TES dimatology}

The main difference between the original retrieval algorithm of aerosol optical depth from THEMS infrered images used by Smith et al. (2003) and the algorithm used in this work is in how the atmospheric temperature profle is obtaned. In the 5 mith et at. 2003) algorithm, concurrent TES observations were used so esimate atmospheric temperatures, but that is no longer possible since the end of systematic Tes spectrometer observations on 31 August 2004 (MY $27, L_{s}=81^{\circ}$ ) Instead, we estmate atmospheric temperatures using a combination of observes THEMS Band 10 temperature and historic TES observations (climatology) from the season and location of interest.

Fig. 7 shows dust aptical depth retrieved using obseved THEMIS Band 10 temperature information compared to that retrieved using TES climatology alone without correction. In the figure, the red points are those observations taken during the 2007 planet-encirding tust storm event (MY $28, L_{s}=265^{\circ}-305^{\circ}$ ), while the black points are those taken at other times. The bitie lines show when the wo dust optical depth results are the same within uncertainties.

As expected, when there are no large dust stoms the difference between retrieved dust optical depth using TES climatology and the correction using THEMIS Band 10 temperatures is essentially withis the uncertainty of the algothm. However, during large dust storms it is necessary to use observed THEMIS Band 10 temperatures to get an accurate retrevat. During large dust storms the observed THEMS Band 10 temperature is higher than the elmatology temperatures from the first year of TES observations (MY 24, 

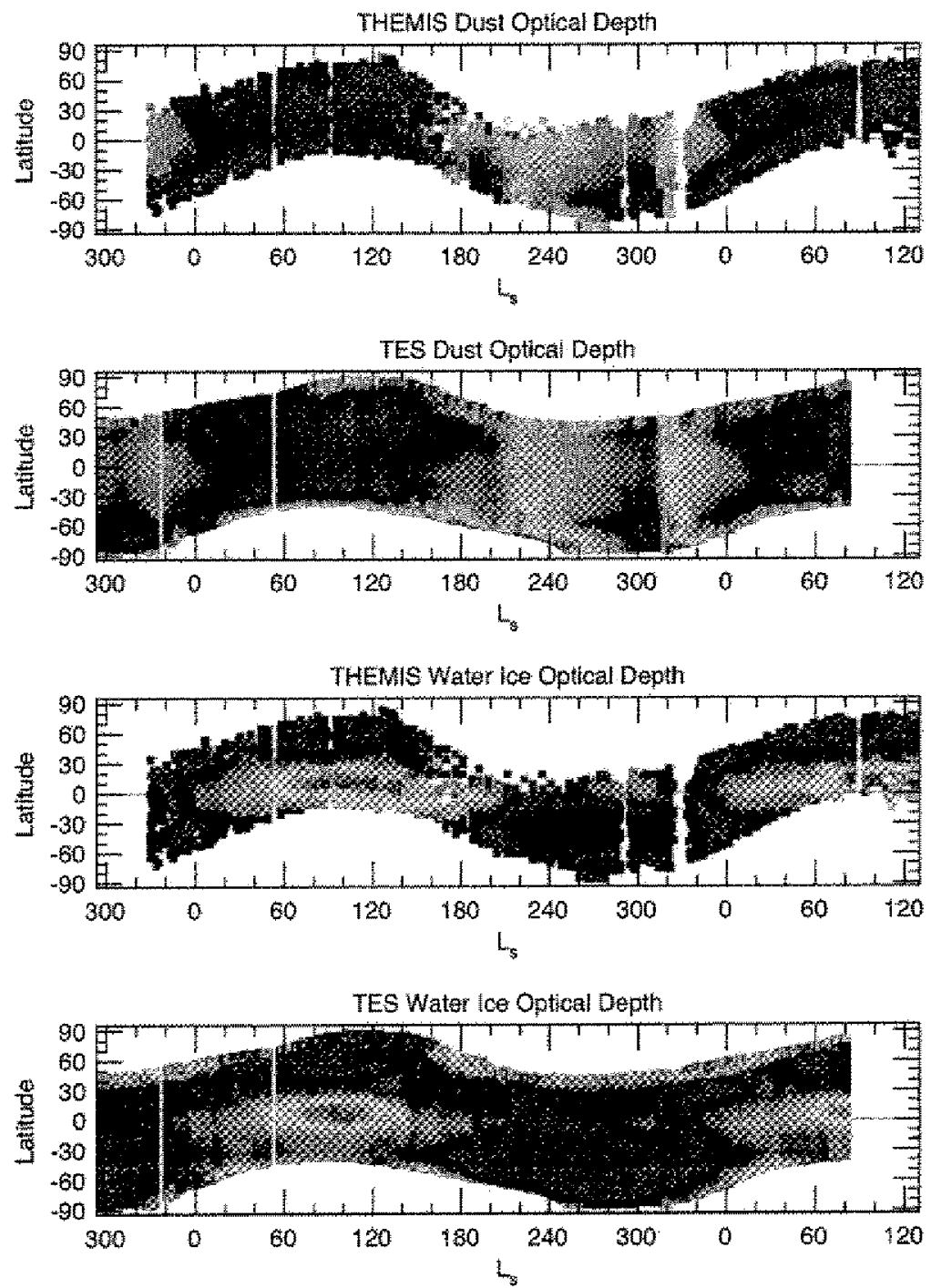

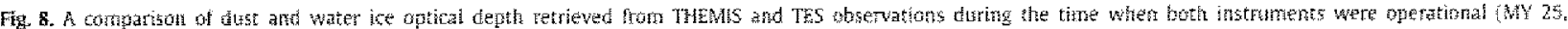

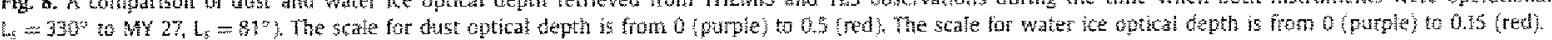

$L=104^{\circ}$ to MY $25, L=104^{\circ}$, which was a very mild year for dust stoms (Smith, 2004). Atmospheric tempezatures greater than cimatology leac to a smafler themal contrast between the surface and atmosphere than would be expected from climatology. Therefore, to produce a spectral feature of a given size, a greater amount of dust must be present that would be required from atmospheric temperaturas from clinatology.

Fig. 7 shows that the above effect can be quite significant during the largest dist stoms: The dust opticat depth can be underestimated by up to a factor of two if observed temperatures are not used. Dust optica depth can also be underestimated by a smaller anount $(10-20 \%$ ) during large regional stoms as shown by the tapward curve of black points at larger values of dust optical depth.

\subsection{Comparison against concurrent the observations}

Regular observations by the TES spectrometer continued for more than a martian year after the beginning of THEMIS observations alowing for a direct comparison beween the two instruments and a valdation of results from THEMIS. Fig. 8 shows side- by-side plots of dust and water ice optical depth retrieved from TES and THEMis, the correspondence between the two is close, with both the seasonal and spatial patterns and the ampitudes of results matching well. Both TES and THEMSS observe the decay of a regional dust stom at the end of MY 25 , the usual low dust optical depth during the aphelion season, and a relatively mild dusty season in MY 26 with moderate regional stoms at $L_{s}=210^{\circ}$ and $315^{\circ}$. The aphelion season water ice cloud belt is observed by both instruments to begin intensification after $L_{s}=0^{\circ}$, reach fult strength at $L_{s}=60^{\circ}$ with the same amplitude and latitude extent, and linger until about $L=180^{\circ}$.

One diffenence is the apparent doudiet conditions observed by THEMIS between $L_{-}=120^{\circ}$ and $180^{\circ}$. This was interpreted by Smith et al. (2003) as a tesulte of the fater local the of the THEMIS observations. OnIy one year (MY 26) in that seasonal period was observed by both TES and THEMIS concurrently. However, observations during this season for othet years (MY 24 and 25 for TES; MY 27, 28, and 29 for THEMS) show that during roughly $\mathrm{L}=100^{\circ}+80^{\circ}$ the water ice optical depth observed by THEMIS is alwavs somewhat higher than that observed by TES, ex- 
cept for the sudden drop in douds observed by THEMS at MY 27 , $L_{s}=135^{\circ}$ at the onset of an unusualy early low-iatitude dust stom, The difference in optical depth is about 0,03 . which is close to the lavel of uncertanly. Nevertheless, the consistency of the trend over several years of observations from both instruments gives support to the idea that cloud optical depth varies with local time, a tendency also observed from telescopes (Wolff et al. 1999 ; Akabane et al. 2002; Glenar et al., 2003), Viking Orbiter (Tamppari et al. 2003). Mars Gobal Surveyor (Wilson et at., 2007), and Mars Recomaissance Orbiter (Malin et al. 2008).

Notable in Fig. 8 is the smaller iatitudinat mange given by the THEMIS observations because of its later local time and thus cooler sufface temperatures. This prevents the THEMIS observations from observing the polar hood water ine clotus seen by TES as well as some of the smater dust stoms along the edge of the polar caps. At the present time the Mars Odyssey spacecraft ts petforming maneuvers to move the local time of the orbit earlier. to about $3000 \mathrm{PM}$. The move is expected to be complete in late 2009. This should provide a somewhat gteater latitude extent for future aerosol optical depth retrievals, and may help provide at wore defontive conclusion about the diumal vartaton of water ice douds in THEMIS observations.

\section{Summary}

Infrared images taken by the THEMS instrument on-board Mars odyssey afe well-sutited for the retrieval of dust and water ice optiGal tepth in the Mars atmosphere. Use of atmospheric temperature infomation contatmed in THEMIS Band 10 data in the retrieval ajgorithm provides a much more accurate retrieval duming large dust stoms as compared to using temperatures from a climatology database alone. During the planet-encircing dust storm event of 2007 (MY 28), the observed THEMIS Band 10 atmospheric temperatwes were $30 \mathrm{~K}$ warmer than those from TES climatology (Smith, 2004). Using a temperature profile based on the observed THEMIS Band 10 temperatures instead of directly from TES timatology resulted in an increase in retrieved dust optical depth durng the stom by a factor of two of noie.

The long and nearly-continuous record of THEMIS retrievals of aerosol optical depth provides a critical link between historic observations from Mars Global Surveyot date and the recent observations by the Mars Express and Mars Reconnaissance Orbiter spacecratt. Compertisons of aerosol optical depth retrieved from THEMS and concurrent TES observations shows strong atgeement providng a validation of the consistency of results from the two instrumetsts. Similar cross-correlation and validation is currently underway comparing THEMIS retrievals agatnst those from Mars Express and Mars Reconnaissance obiter instruments (e.g. Smith et at., 2009; Woiff et al, 2009).

Over a time span of more than 3.5 martian years, THEMIS observations have shown both repeatable patterns and large inteannual variation in aerosol jehavior. Mars Years 26 and 27 were observed to have moderate dust activity during the perihelion (southem spring and summer) season, with regiond dust stoms near $b_{5}=220^{\circ}$ and $315^{\circ}$, while MY 28 featuted a planet-encircling dust storm event just before southem summer solstice but no lateseason regional storm at $\mathrm{L}_{\mathrm{s}}=315^{\circ}$. Early season low-latitude dust stoms were observed in both $M Y 27$ and 29 before $L_{s}=180^{\circ}$, a type of evert not observed by TES during MY $24-26$.

The low-latitude aphelion season water ice doud belt was observed each year from MY $26-29$ to begin forming at $1_{s}=0^{\circ}$, reaching peak optical depth between $\mathrm{L}_{s}=100^{\circ}$ and $120^{\circ}$, and gradually decay after $L_{s}=140^{\circ}$. Clout thickness was observed to rapidy decline durng the early-season dust stoms in MY 27 and 29, and to recover to nominal values after dissipation of the dust storms.
The limited fatitudinal extent of reliable THEMIS retrievals be cause of the late locat time $(-5: 00$ PM) of the Mars odyssey orbit preduced the observation of both polar hood clouds and small cust storms along the edge of the polar caps. However, the later local time of THEMS observations compared to TES ( $2200 \mathrm{PM})$ does allow for a limited exanination of the variation of cloud op tical depth with time of day. The THEMIS observations consistenty show somewhat tigher water ice optical depth in the low-latitude aphelion season cloud belt, especially after $\mathrm{L}=100^{\circ}$.

The THEMIS instrument remains fully operational and contmues to add to its long bistory of atmospherie observations. The Mars Odyssey spacecraft is currenty tandergoing maneuvers to bring the local time of its oftet to an earlier time. It is expected that the ma. neuvers will be completed in late 2009 with the orbit neal $3: 00$ PM local time. observations at the earliet time will allow the retrieval of aerosof optical depth over a greater latitude range and will provide further information on the time-of day variation of water ice optical depth.

\section{Acknowledgments}

We thank the THEMIS opertions team at Arizona State University for their expent accuisition, calibration, and handing of THEMIS data, and Phin Christensen for his continued support of THEMIS atrospheric observations and analysis. We thank Josh Bandfeld for useful discussions about the THEMIS data and retrievals, and Michael Wolf and an anonymous referee for their helpful comments on this manuscript. This work was stpported through the Participating Scientist program of the NASA Mars odyssey project.

\section{References}

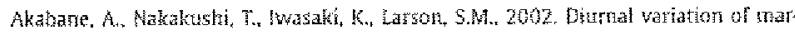

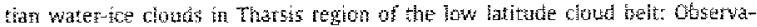
fion in $1995-1909$ apotrtions. Astren. Astaphys. 384, $678-688$.

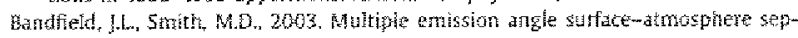
aration of themal emission spectrometer data, learys $661.47-65$.

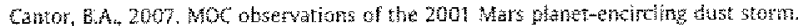
haris $180,60+30$

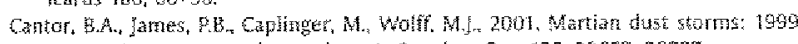
Mars orbiter camera observations. J. Geophys, Res. $106,23653-26687$,

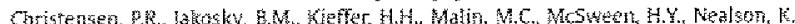

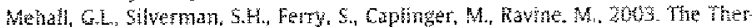

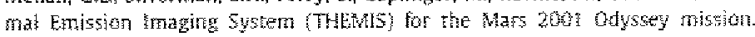
Space Sci. Few 110, $85-130$.

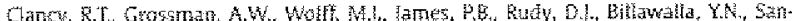

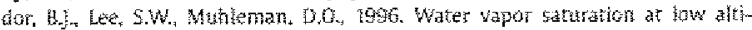

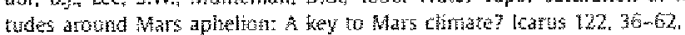

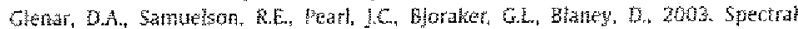
imaging of pantian water ice clouds and their bituna behwion durng the 1990 aphelion season. lcarus 16:, $29 \%-318$.

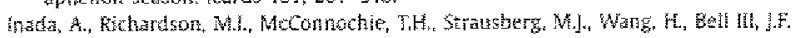

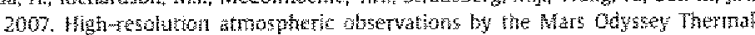

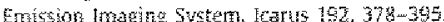

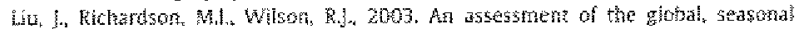
and intermmial seracecaft tecord of martian climate in the thermal infrareat

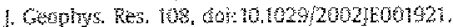

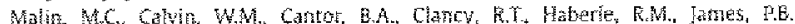

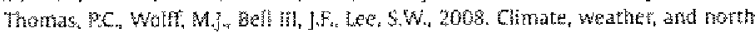

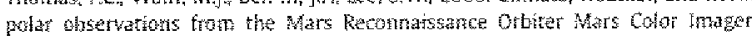
catus $194,501-572$.

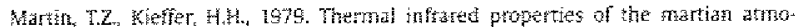

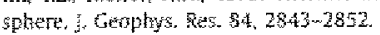

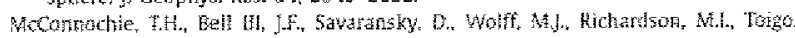

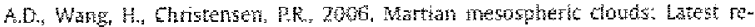

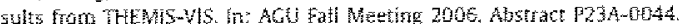

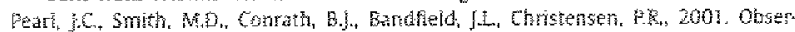
vations of martan ice clouds by the Hars Gobal Sunver Thernal Emision

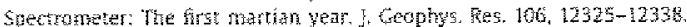

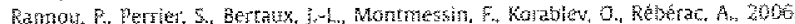

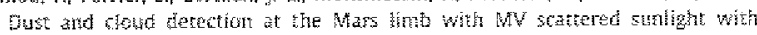

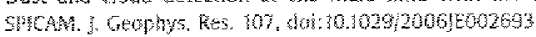


Smith, M.D. 2004 Interandud variability in TES anosphenic observations of Mars during $1599+2007$, Icarus $167,148-165$.

Smith, MD. 2008. Spacecraft obserwatigns of tre matian atmasphere. Annu, Rev Earth Pianet. 50 . $36.191-219$.

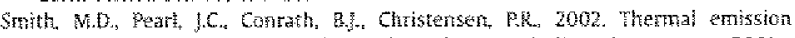
spectronetef bbervations of mattian planet-encichn Gustorm 200 a. Lats $157,259-263$.

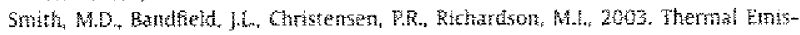

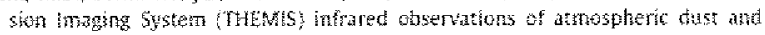

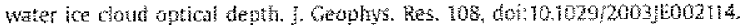

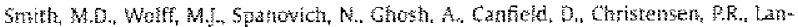

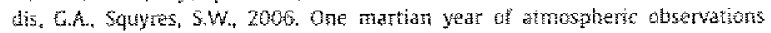

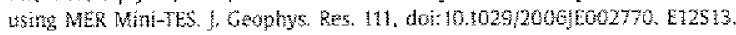

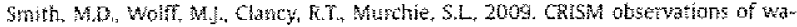
ter yapor and cabon monoxidu. J. Geophys. Res. dat 101029) press.

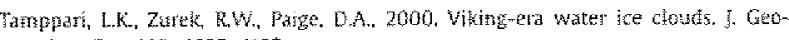
plys. Kes. 10s, $4087-470$ ?

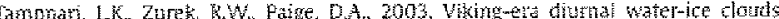

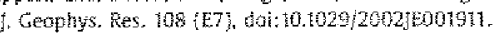

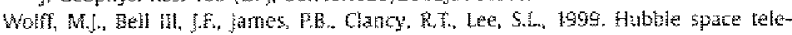

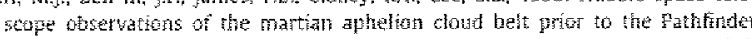
mission: Segsonal and isterantual vanations. Geophys Res 104 (E4) $907-5041$.

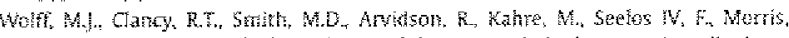
F.V. 2009. Wavelergh dependence of dust aerosol single scattering albouo as

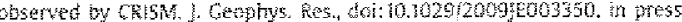

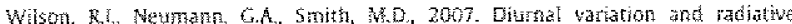

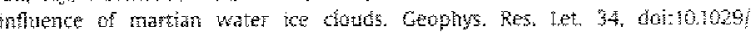
20065027976.102710 .

Zasou, $h$ and 20 colleatues. 2005. Water touds and bust aerosos peservations with PPS MEX at Mors Phant Space Sed. 53, $1065-1077$ 